\title{
A Study on the Bridging Strategy between the Theory of Fluid Mechanics and Engineering Applications

\author{
Guoquan Xiao*
}

\author{
School of Mechanical \& Automotive Engineering, South China University of Technology, \\ Guangzhou, China, 510641 \\ ${ }^{*}$ Corresponding author
}

Keywords: Fluid mechanics, Bridging teaching strategy, CFD engineering application.

\begin{abstract}
Aiming at the problems existing in the teaching of fluid mechanics theory, this paper puts forward the goal of introducing CFD (Computational Fluid Dynamics) software teaching, Combining with the goal, this paper discusses the bridging strategy between fluid mechanics theory and CFD Engineering application: (1) Motivate students using visual display of flow phenomenon close to life or engineering practice; (2) Deepen understanding of concepts and principles based on the CFD solution process; (3) Deepen understanding of basic equations and their applications through the combination of "digital shape" to expound the actual fluid equations and their applications; (4) Improve experimental content and methods through CFD application; (5)Promote undergraduate seminar teaching; (6) Promote CFD technical engineering application.
\end{abstract}

\section{Introduction}

Fluid mechanics is a compulsory course for undergraduates majoring in mechanical, energy, civil, construction, and chemical engineering[1,2].The knowledge point has a large capacity, and the concepts and principles are abstract and boring, but they are closely integrated with the engineering practice. Traditional theoretical teaching combined with CFD software training is difficult to truly understand the theoretical knowledge and movement laws of fluids, and it is difficult to really use CFD software to develop products[3,4].

In order to improve the teaching effect of fluid mechanics theory, the application of CFD software is effective to promote students to understand the abstract flow phenomenon, familiar with CFD software engineering application process and method, and explore the bridging teaching method of fluid mechanics theory teaching and CFD software engineering application.

\section{Introducing the Goal of CFD Teaching}

Fluid mechanics includes basic concepts, principles and equations, dimensional analysis, interdisciplinary content, and penetration and application of engineering practice. Although theoretical teaching can use dynamic display courseware made by flash, 3dmax, etc.[5], it is difficult to concentrate the shortcomings of students' attention by overcoming the monotonous expression[6,7]. However, such materials lack the organic combination with the actual model, only qualitative demonstration, can not achieve the effect of quantitative data specific analysis[8]. The objectives of introducing CFD teaching are: (1) Motivate students with flow visualization; (2) Deepen understanding of concepts and principles with CFD software commands; (3) Deepen understanding of basic equations and their applications through CFD solutions; (4) Improve experimental content and methods through CFD application; (5) Promote undergraduate seminar teaching; (6) Promote CFD technical engineering applications.

\section{The Exploration of Fluid Theory and CFD Software Engineering Application}

\section{Motivate Students with Flow Visualization}

Fluids include two states in the "three states" of nature, which are closely related to daily life. The 
fundamental attribute of fluids to solids is fluidity. Fluids are easily deformed and easily compressed. Gases are invisible and invisible. Fluid mechanics knowledge is abstract and difficult to stimulate students' interest.

In the classroom teaching, the CFD simulation is introduced to give the visual results to be close to the actual life or engineering phenomenon, such as the weather forecast and the train crosswind visualization results are shown in Figure 1[9] and Figure 2, respectively.

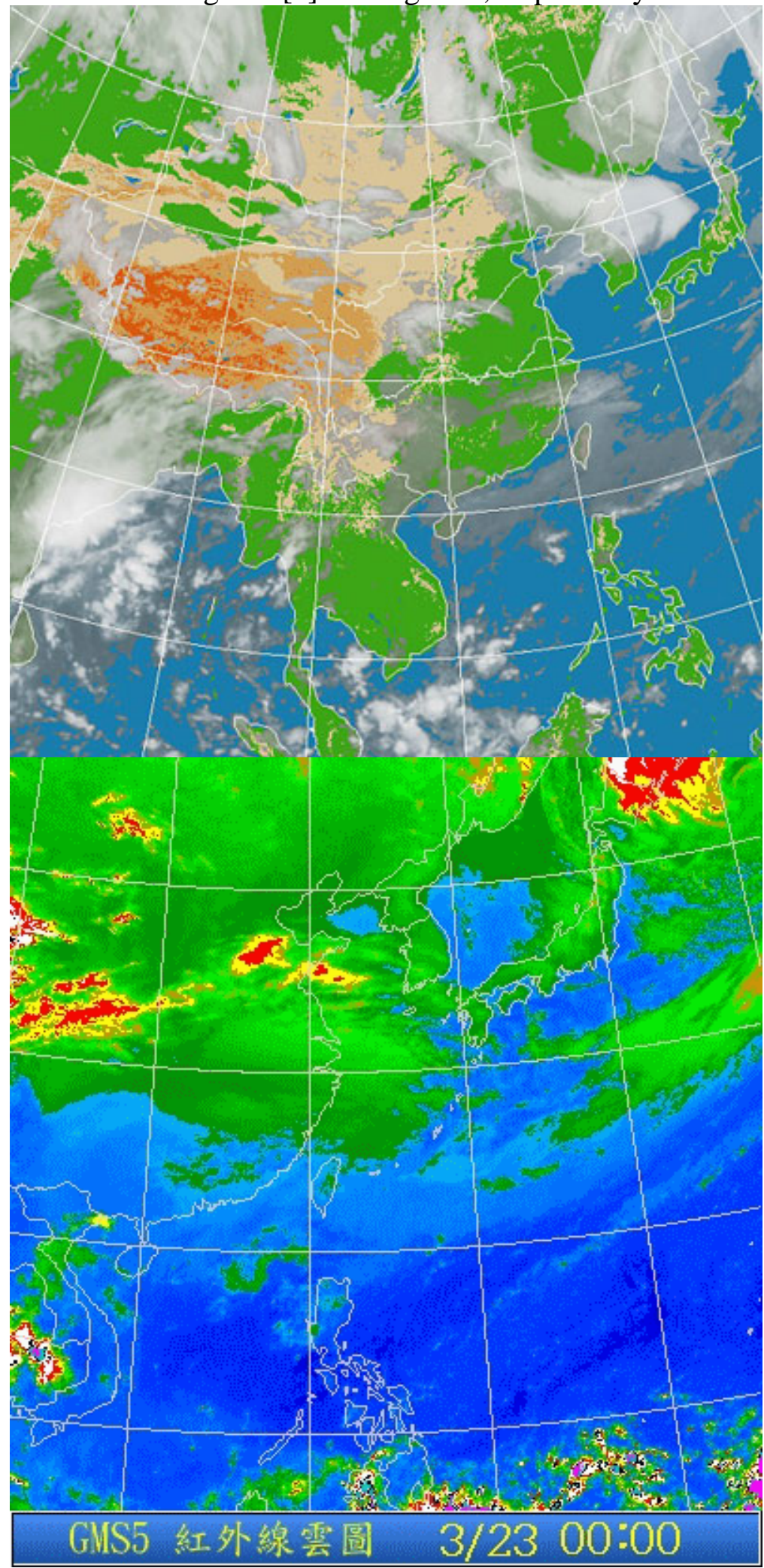

Figure 1. Weather forecast 


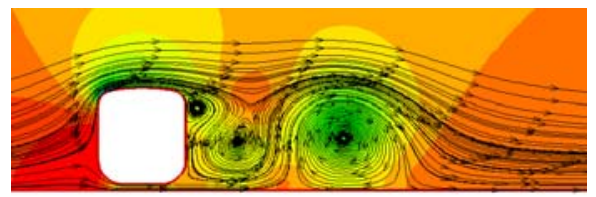

Figure 2. Train crosswind phenomenon

Through visual demonstrations, students are considered to be a "fascinating" course from the very beginning. At the same time, it can also enable students to understand the real needs in life or engineering, and inspire students to learn the basics of fluid mechanics and CFD methods to meet this demand, improve students' ability to master knowledge, and apply what they have learned.

\section{Deepen Understanding of Concepts and Principles with CFD Software Commands}

The concepts and principles of fluid mechanics are abstract and difficult for beginners to understand. Combine the choice of window commands in the CFD problem solving setup to understand the important concepts used, such as steady and unsteady flow, compressible and in-compressible, spin and spin, laminar flow, turbulence model, basic physical parameters (density, viscosity, etc. ), initial and boundary conditions, etc[10].The introduction of CFD in the theoretical teaching all the words in the software and the concepts scattered in the various chapters of the textbook are all helpful to the students to understand and understand these concepts as a whole, and to understand and memorize.

At the same time, in the classroom, CFD can be used to solve the post-processing, and the threedimensional full flow field (speed and temperature, etc.) information can be visually presented to the students in the form of images, as shown in Figures 3-4.

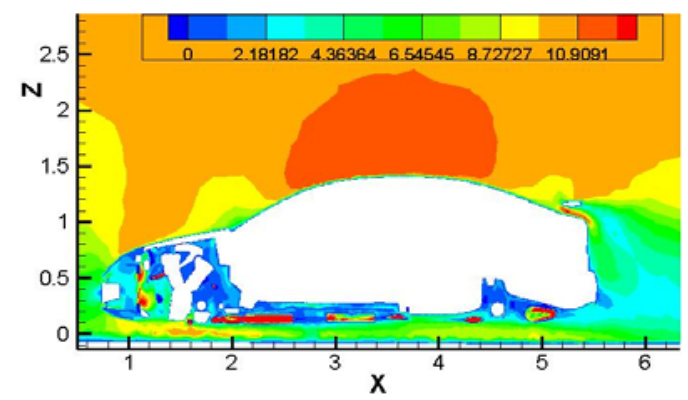

Figure 3. Speed distribution of a car

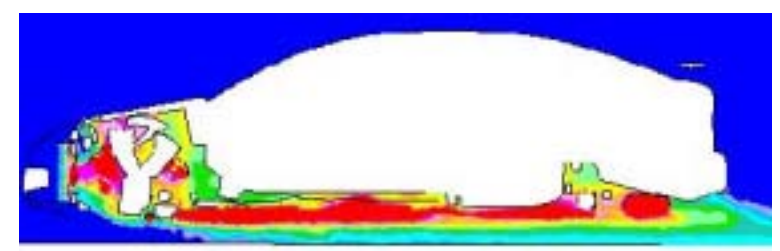

Figure 4. Temperature distribution of a car thermal management system

These images help to understand the velocity field, temperature field, etc., overcome the abstraction of the concept, and deepen the understanding and application of the concept.

\section{Deepen Understanding of Basic Equations and Their Applications through CFD Solutions}

Methods for describing fluid motion are the Lagrangian method and the Euler method. The Lagrangian method tracks the trajectory of the fluid particle, the physical meaning is clear, the equation is easy to derive, and the model is simple, but it is difficult to obtain the analytical solution in practical application; the Euler's law introduces the concept of the field, and establishes the model from the mathematical point of view. Numerical method is solved. For example, when Euler's method is described, a pipe model is introduced as an example to introduce a control body to establish a mathematical model, as shown in Figure 5. 


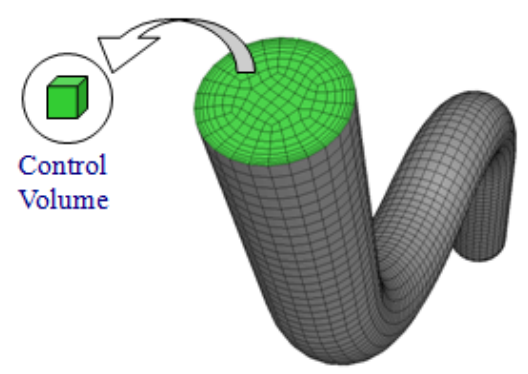

Figure 5. Schematic of the tube flow grid

Based on the control volume to the establish fluid equations of the tube flow, as shown in (1), the conservation of mass, momentum, energy, etc. is respectively solved.

$$
\frac{\partial}{\partial t} \int_{\mathrm{V}} \rho \phi d V+\oint_{\mathrm{A}} \rho \phi \mathbf{V} \cdot d \mathbf{A}=\oint_{\mathrm{A}} \Gamma \nabla \phi \cdot d \mathbf{A}+\int_{\mathrm{V}} S_{\phi} d V
$$

In (1), the first and second terms on the left are unsteady and convective, respectively, and the first and second terms on the right are diffusion and source, respectively. ${ }^{\phi}$ is the flow of physical quantities, $\Gamma$ is the diffusion coefficient, ${ }^{S_{\phi}}$ is the source item. When ${ }^{\phi=1, \Gamma_{\phi}}=0$,(1) is a continuity equation; when

$\phi=u, v, w, \Gamma=\mu(1)$ is respectively Momentum equation of direction; When ${ }^{\phi}=k, \varepsilon, \Gamma=\mu_{e} / \sigma_{k}, \mu_{e} / \sigma_{\varepsilon},(1)$ is respectively

$k-\varepsilon$ Equation; when ${ }^{\phi=C_{p} T, \Gamma=\mu_{e} / \sigma_{h}},(1)$ is the energy equation.

The above-mentioned problem of actual tube flow is combined by "digital form". On the one hand, the concept of the control body is deepened, and more importantly, it can give a convenient analysis in mathematics, which is conducive to understanding the concept from a mathematical point of view. On the other hand, the Euler equation-based continuity (mass conservation) equation, the Euler (momentum conservation) equation of the ideal fluid, and the Bernoulli (energy conservation) equation are all scattered in the various chapters of the textbook to effectively solve the problem. Students understand and understand the difficulties of fluid equations as a whole, and deepen their understanding of the relationship and difference between fluid equations. They also deepen the equation by setting the CFD calculation model, the realization of equations and the convergence judgment and post-processing results. The understanding of the group application.

\section{Improve Experimental Content and Methods through CFD Application}

There are three main ways of fluid mechanics experiment teaching: (1) relying on sophisticated equipment and the complicated operation of the experiment teacher to demonstrate the real flow, it is difficult for undergraduates to participate in the whole process, and the intuitive feeling is lacking; (2) the operation is relatively simple but the number of devices Classical experiments with great dependence, such as the "tube laminar flow / turbulence" experiment.(3) Virtual experiments based on Flash and Web platform development, this experiment is derived from "man-made", too "virtual", usually only the results of experiments, lack of process explanation to achieve such results, the authenticity of the experiment will be subject to students The doubts, as well as the content of lectures in the classroom, the amount of information (especially virtual experiments), students often have no time to think and ask questions carefully. The introduction of CFD numerical experiments is essentially based on the basic principles and equations of fluid mechanics to simplify the actual engineering problems, and select the appropriate mathematical model to solve, including the process of understanding phenomena, analyzing problems, comparing methods and judging results. This kind of CFD numerical experiment can replace some classical experiments, and can also update the experimental content and methods. It can supplement and expand the traditional fluid teaching with insufficient experimental conditions, which helps students understand the complex flow phenomenon of the change process. Students organically combine mathematical equations, CFD 
software, and physical issues to enhance understanding of the physical nature of the flow process. For example, in the external flow problem often encountered in vehicle development, the boundary layer appears separated by the structural modeling, meshing, and unsteady simulation, and alternate vortices appear downstream of the cylinder under the mainstream driving. Forming the Karman vortex street and the tail vortex area, the process is made into an animation, as shown in Figure 6.

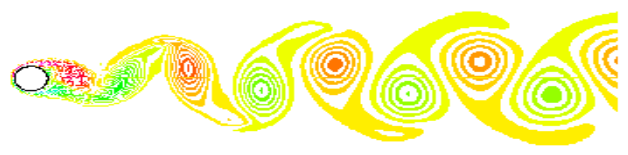

Figure 6. Cylindrical flow around the Carmen vortex street

Through realistic simulation of animation, students can visually see the changes in the flow field during the flow, thus deepening the understanding and mastery of the problem. In other words, the low-cost, high-efficiency cfd numerical experiment can enrich the experimental content and improve the experimental teaching method.

\section{Promote Undergraduate Seminar Teaching}

At present, the undergraduate computer has a solid foundation and is proficient in software. The initial model established by beginners when cfd is used to solve the flow problem in fluid teaching has to be repeatedly confirmed: whether the physical model is correct, whether turbulence, unsteadiness, and compressibility are properly considered. Or three-dimensional effect; whether the boundary condition is reasonable, the calculation domain, the boundary type and the initial value rationality; whether the mesh is sufficient, whether the solution is independent of the mesh, the accuracy of the adaptive mesh is improved a lot, and whether the resolution of the boundary needs to be improved. These problems can stimulate students to participate in teaching interactions, combine the occurrence and development of mobility problems to balance the development of seminar-based teaching, and cultivate students' habits and abilities of independent thinking.

\section{Promote CFD Technical Engineering Application}

Currently, CFD has been widely used in the enterprise product design process.

Although students are familiar with the physical characteristics of products after entering the company, the necessary operation skills for CFD software through simple training are not a problem, but it is difficult to fully understand the nature of flow in the face of actual flow analysis. Using lowcost and high-efficiency CFD numerical solution for flow problems, we can try to solve the problem in multiple angles and directions, and also change the boundary conditions or initial conditions to solve the results under more conditions, so as to cultivate undergraduate research ideas and Engineering concepts, training undergraduates to develop habits that are good at trying and exploring the rules. On the basis of comprehensive grasp of the theory of fluid mechanics and basic equations, the system accumulates the experience of CFD solving the actual flow problem, obtains more accurate simulation results, and truly uses CFD software for product development services.

\section{Summary}

The introduction of CFD teaching in the course of fluid mechanics, combined with the focus of the discussion on the bridging strategy between fluid mechanics theory and CFD engineering application: (1) using the visual display of the flow phenomenon close to life or engineering practice to stimulate learning interest; (2) based on CFD The solution process fully talks about the concept and principle of fluids, and deeply understands and can memorize deeply; (3) For the actual tube flow problem, through the combination of "digital shape", the continuity (mass conservation) equation and ideal based on Euler equation The Euler (Momentum Conservation) equation of the fluid and the Bernoulli (energy conservation) equation are all discussed in order to deepen the understanding of the fluid equations and the differences; (4) Improve the experimental content and methods; (5) 
Conduct research-based teaching, assist Undergraduates participate in engineering practice; (6) promote CFD technical engineering applications.

This article aims to attract more teachers and CFD engineers to jointly promote the teaching reform of fluid mechanics courses and promote the application of CFD engineering.

\section{Acknowledgment}

This research was financially supported by the 2018 South China University of Technology Schoollevel Teaching and Research Teaching Reform Project (Y1180521) and Science and Technology Planning Project of Guangzhou (201802020031).

\section{References}

[1] B.K.Li, W.J. Rong, Z.Q. Liu, and L.M.Li, "Discussion on the introduction of CFD engineering case in the teaching of engineering fluid mechanics," J. Mechanics and Practice, Beijing, vol.40, pp. 93-95, Jane 2018.

[2] Q. Li, D.H.Zhang, W.W.Xu, C.J.Zhou, "The practice and exploration of the teaching reform of computational fluid mechanics course based on the cultivation of engineering practice ability," Higher Education in Chemical Industry, Shanghai,vol.34, pp. 36-40, Jane 2017.

[3] Z.M. Liu, Y.F. Xue, Exploration and Practice of Teaching Reform and Innovation in Fluid Mechanics, J. Green Science and Technology, vol.21, pp. 206-208, 2017.

[4] B. Huang, Q. Chen, Q. Wu, G.Y.Wang, “Analysis of the construction of a fine research-based course based on the OBE concept-Taking the principle and application of thermal fluid computing software as an example,” Science And Teaching journal (mid-year),pp.38-39 s.44,September 2018.

[5] P.Yu, H.F. Guo, “Application of fluent software in engineering fluid mechanics teaching," Education and Teaching Forum, vol.16, pp. 271-272, April 2018.

[6] C.L. Xie, L.Y. Ni, Application and Practice of Computational Fluid Dynamics in Engineering Fluid Mechanics Course, J. Mechanics and Practice,vol.39, pp. 503-505+495, 2017.

[7] W.Shen, J.Yao, X.X.He, X.Xu, "Combination of engineering fluid mechanics and CFD software, innovative practice platform,” J. China Modern Education Equipment, pp. 46-48, March 2017.

[8] D.Q.Zhou, L.W.Zhong, W.D.Liu, Y.Zheng, “Application of CFD numerical test in the teaching of fluid mechanics,” J. Education and Teaching Forum, vol.30, pp. 194-196, August 2017.

[9] W.H.Peng, H.X.Zhang, Application of CFD software in fluid mechanics teaching, J.AsiaPacific Education, vol.7, pp.69, August 2016.

[10] Information on https://image.baidu.com/search/detail

[11] L.Lu, Y.B.Yu, Z.N.Mi, W.L.Li, J.Li. "Reflections on the application of CFD flow field simulation virtual experiment in the teaching of Fluid Mechanics course.” Education and Teaching Forum, vol.13, pp.223-224, 2018. 